\title{
Diagnosis and Treatment Methods for Ocular Surface Disease in Glaucoma
}

\author{
Gemma C M Rossi
}

Professor, University Eye Clinic of Pavia and Medical Doctor, Eye Clinic of the IRCCS Policlinico San Matteo Foundation, Pavia, Italy

\begin{abstract}
Glaucoma and ocular surface disease (OSD) are often concomitant disorders (OSD is the consequence of a tear film dysfunction that may be due to several conditions). The aims of the paper are to suggest a sequence of diagnostic tests easy to perform in daily practice, both subjective and objective, to detect the onset of the OSD; and, second, to propose how to prevent and, if the case, how to manage the OSD. Briefly, tear film break-up time (TF-BUT) and corneal/conjunctival staining are suggested to detect the main signs of OSD. In terms of therapy, the long-term exposure to benzalkonium chloride (BAK) should be minimised, preferring non-BAK-preserved or BAK-free glaucoma medications, where available, as well as fixed combinations. The treatment of associated diseases of the anterior surface may reduce signs; use of non BAK-preserved tears may reduce symptoms.
\end{abstract}

\section{Keywords}

Ocular surface disease, glaucoma, BAK, preservative-free, TF-BUT, corneal staining, glaucoma symptom scale

Disclosure: Gemma C M Rossi has no conflict of interest to declare. No funding was received in the publication of this manuscript. Received: 6 April 2014 Accepted: 22 May 2014 Citation: European Ophthalmic Review, 2014;8(1):40-3 DOI: 10.17925/EOR.2014.08.01.40 Correspondence: Gemma Caterina Maria Rossi, IRCCS Policlinico San Matteo Foundation, P.le Golgi 19, Pavia 27100, Italy. E: gemma.rossi.md@gmail.com

Glaucoma is a chronic disease of the optic nerve, often associated with elevated intraocular pressure (IOP), which can cause an irreversible Ioss of vision. ${ }^{1}$ IOP remains the most clinically important risk factor for developing and/or progressing glaucoma. In fact, international randomised clinical trials have demonstrated that reducing IOP can slow the progression of disease in patients with glaucoma (Advanced Glaucoma Intervention Study [AGIS] in 2000;" Early Manifest Glaucoma Trial [EMGT] in 2003²), and can delay or even prevent the development of open-angle glaucoma in patients with ocular hypertension (Ocular Hypertension Treatment Study [OHTS] in 2002; European Glaucoma Prevention Study [EGPS] in 20054).

IOP reduction is most commonly achieved using topical ocular medications; 5 but chronic topical IOP-lowering therapy is associated with chronic exposure to vehicle components, in particular to preservatives, employed to maintain stability and sterility of the product.

Preservatives can be associated with undesirable adverse effects such as allergy, local irritation and inflammation. ${ }^{6}$

Benzalkonium chloride (BAK) is the most commonly utilised preservative in ophthalmic preparations and also in IOP-lowering medications. Its effects on the ocular surface have been studied extensively both with in vitro and with in vivo clinical studies. ${ }^{7-9}$ BAK induces apoptosis and increases concentrations of inflammatory markers not only at corneal and conjunctival epithelial layers but also at trabecular meshwork and non-pigmented ciliary epithelial cell lines. ${ }^{10}$ Clinical studies have demonstrated its effects on increased corneal staining and decreased tear break-up time (TF-BUT). ${ }^{8,11-13}$

Therefore, it should not be surprising to detect glaucoma and ocular surface disease (OSD) as concomitant disorders, where the term 'OSD' is a general and not causal diagnosis of the non-specific symptoms associated with irritation of the conjunctival and corneal surface.

OSD, in fact, is the consequence of a tear film dysfunction that may be due to several unrelated causes and conditions, such as age, systemic diseases (autoimmune disorders, diabetes, Sjögren's syndrome), ocular diseases (blepharitis, lid margin irregularities, contact lens use, ocular surgery, refractive surgery), hormonal changes (menopause), topical (toxic drugs, preservatives) and systemic drugs (see Figure 1). ${ }^{14}$

The tear film is fundamental in the maintenance of the ocular surface: alterations in quantity and/or quality result in an unstable tear film and in the onset of an irritation to the front of the eye that may lead to sign and symptoms of OSD.

The prevalence of OSD lies in the range of $5-30 \%$ of the population aged 50 years and older; ${ }^{15}$ the Beaver Dam cohort study found that the overall prevalence was $14.4 \%$, ranging from $8.4 \%$ in subjects younger than 60 years to $19.0 \%$ in those older than 80 years. ${ }^{16}$

Glaucoma patients are therefore at risk of developing OSD for two reasons:

1. open angle glaucoma (OAG) is typically a disease of the elderly: the prevalence of OAG in people over 40 years is $2.1 \%$ (95\% confidence interval [Cl] 1.7 to 2.5$)$, and ranges from $0.3 \%(95 \% \mathrm{Cl} 0.1$ to 0.5$)$ in people aged 40 years to $3.3 \%$ (95\% Cl 2.5 to 4.0 ) in people aged 70 years; ${ }^{5}$ and

2. glaucoma patients are usually chronically treated with preserved topical drugs: the prolonged use of preserved topical drugs causes an increased tear evaporation, inducing an immunological and/or toxic response from the ocular surface. ${ }^{6}$ In glaucoma patients over 40 years old, BAK-linked effects usually combine with age-related dry eye. 
Figure 1: Dysfunctional Tear Film Pathogenesis and Maintenance

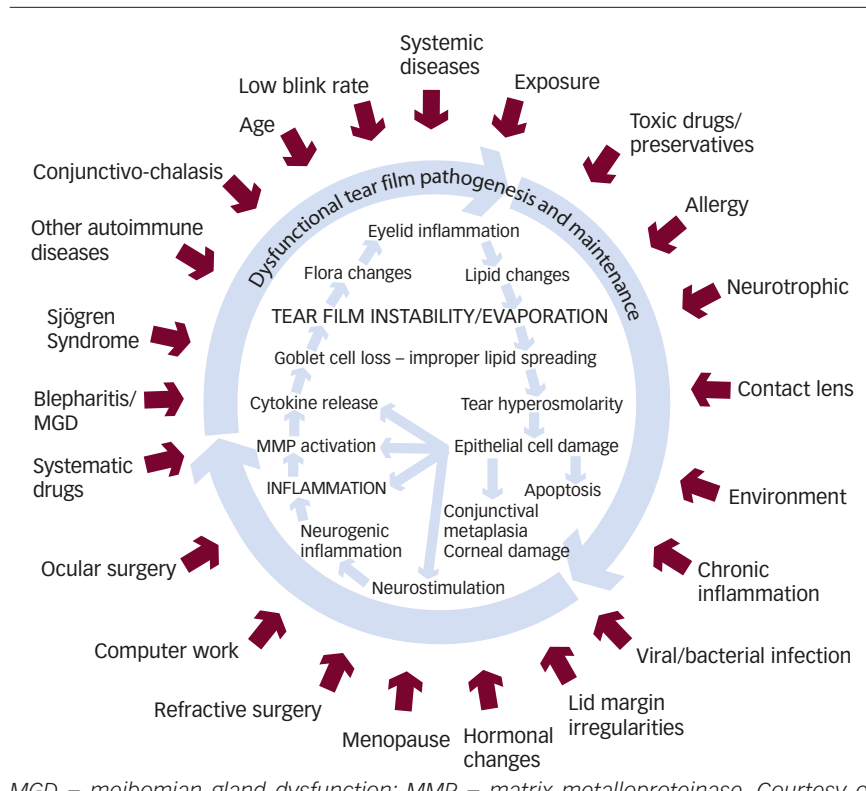

$M G D=$ meibomian gland dysfunction; $M M P$ = matrix metalloproteinase. Courtesy of Professor Rolando.

Some papers, in recent years, have investigated the prevalence of OSD in glaucoma patients, detailed below.

In 2006, Tsai and colleagues observed that the prevalence of glaucoma in patients with severe OSD was $66 \%{ }^{17}$ Leung and co-workers in 2008 found that $59 \%$ of glaucoma patients reported symptoms of dry eye. ${ }^{11}$ Rossi in $2009^{12}$ and Fechtner in $2010^{13}$ found a high prevalence of OSD among medically treated glaucoma patients ( $27.8 \%$, but $40 \%$ in patients taking two/three drugs and $48 \%$, respectively). Therefore, a large proportion of patients with open-angle glaucoma or ocular hypertension had signs and/or symptoms of OSD in at least one eye: but how to diagnose them?

In fact, although a variety of diagnostic tests are in common clinical usage, there is no consensus on which combination of tests should be used to define the disease, either in the clinic or for the purposes of a research protocol.

Moreover, it must be remembered that a poor correlation has been found between objective and subjective signs of dry eye disease ${ }^{18,19}$ underlining that patient's self-assessment of dry eye severity may differently relate to objective signs.

We suggest an easy sequence of ophthalmic examinations that should be performed to glaucoma patients to point out ocular surface alterations in the clinical practice.

\section{Diagnosis}

\section{Sequence of Diagnostic Tests}

The diagnosis of OSD is based both on symptoms referred by patients (history and 'dry eye questionnaires') and on signs recorded by the ophthalmologist (objective tests).

\section{Symptoms Tests}

Symptoms reported by glaucoma patients can be collected and have been used to diagnose the condition in several previous studies; however, the use of a questionnaire to assess symptoms and their

\section{Figure 2: The Glaucoma Symptom Scale Questionnaire (English Version)}

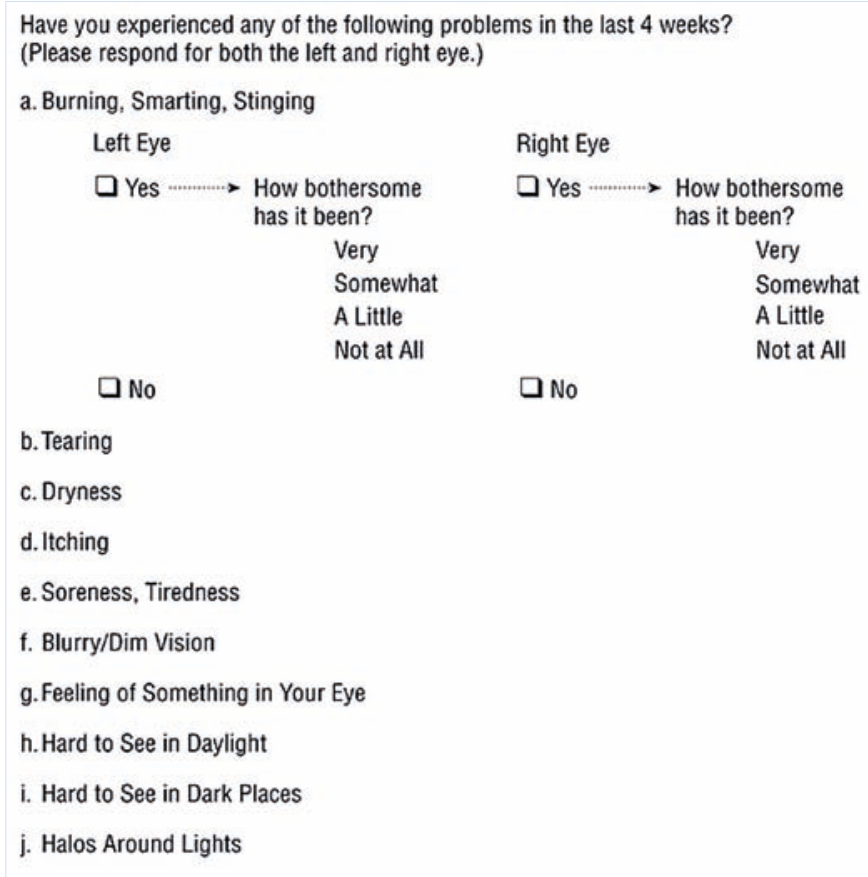

Figure 3: Reduced Break-up Time

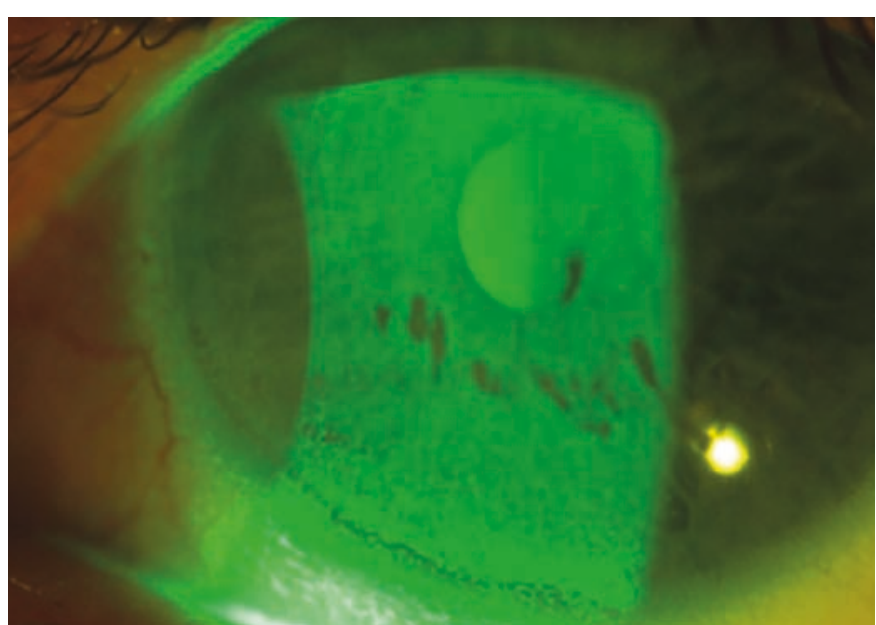

impact on vision-related function should be preferable since the answers are standardised and comparable over time.

A number of instruments have been developed to understand patients' experience of the OSD and its impact on their quality of life (QOL). A few glaucoma patient-specific questionnaires have been developed..$^{20,21}$

To be routinely adopted in the clinical practice a questionnaire must be easy and fast to complete; for these reason, we suggest that the Glaucoma Symptom Scale (GSS) should be used.

The GSS ${ }^{21}$ is a disease-specific questionnaire used to quantify the specific impact of glaucoma either on visual ocular complaints or non-visual ocular symptoms (see Figure 2). The questions refer to a 4-week recall period. It includes a function subscale, a symptoms subscale and an overall average score. All subscales may be evaluated as the average of both eyes or separately for each eye. 


\section{Figure 4: A) Fluorescein Corneal Staining; B) Conjunctival Lissamine Green Staining}
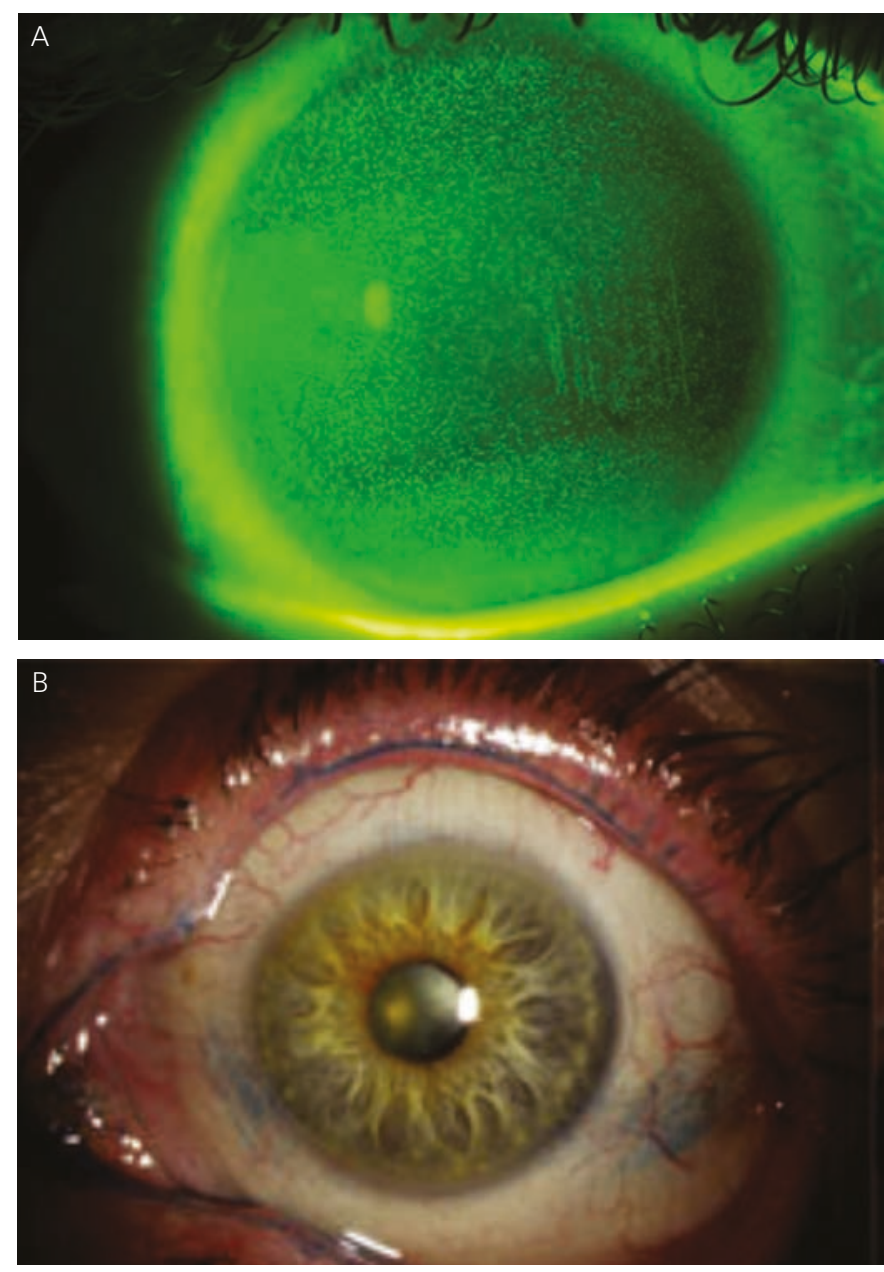

Figure 5: Oxford Scheme Grading

Griteria
$\begin{aligned} & \text { Equal to or less than } \\ & \text { panel A }\end{aligned}$

The questionnaire has been shown to discriminate between persons with and without glaucoma, and is available in English, Spanish and Italian versions. ${ }^{21-23}$
Table 1: Anti-glaucoma Medications Available Benzalkonium Chloride-free

\begin{tabular}{lllll} 
Generic & Brand & Company & Location & Dosing \\
Apraclonidine & Iopidine & Alcon & EU & Unit \\
\hline Betaxolol & Betoptic S & Alcon & US/EU & Unit \\
\hline Bimatoprost 0.3 & Lumigan 0.3 & Allergan & EU & Unit \\
\hline Brimonidine purite $\left.{ }^{*}\right)$ & Alphagan P & Allergan & US & Multi-dose \\
\hline Carteol & Several & Sevaral & EU & Unit \\
\hline Dorzolamide & Trusopt & Merck & EU & Unit \\
\hline Dorzolamide/Timolol FC & Cosopt & Merck & EU & Unit \\
\hline Pilocarpine & Several & Several & EU & Unit \\
\hline Pilocarpine/Timolol FC & Fotil & Santen & EU & Unit \\
\hline Tafluprost & Taflotan & Santen & EU & Unit \\
\hline Tafluprost & Saflutan & Merck & US & Unit \\
\hline Timolol & Several & Several & US/EU & Unit/Multi-dose \\
\hline Travoprost BAK-free & Travatan Z & Alcon & US & Multi-dose \\
\hline Travoprost with polyquad $\left({ }^{*}\right)$ & Travatan & Alcon & EU & Multi-dose \\
\hline
\end{tabular}

*alternative preservative system. BAK = benzalkonium chloride;

$F C=$ fixed combination

\section{Signs}

OSD is diagnosed by objective methods that examine different aspects: tear flow (Shirmer I and II, evaporimetry, fluorimetry, dye dilution, etc.); tear stability (break-up time [BUT], symptomatic BUT [SBUT], TF-BUT, fluorescein, non-invasive BUT [NIBUT], tear film stability analysis system, etc.); and tear composition (lactoferrin, lysozyme, peroxidase, immunoglobulin A, impression cytology, flow cytometry, confocal microscopy, interferometry, osmolarity, tear ferning, grading staining, fluorescein stain, Rose Bengal stain, lissamine green, etc. $)^{15}$

In my opinion, the following examinations can be easily performed by all ophthalmologists in daily practice to all glaucoma patients with no adjunctive time or costs to the standard visit: the fluorescein TF-BUT and the corneal and conjunctival staining (fluorescein and/ or lissamine).

The TF-BUT method assesses tear film stability. TF-BUT values greater than or equal to 10 seconds are coded as normal and values less than 10 seconds as abnormal. The fluorescein break-up time is usually performed prior to grading (see Figure 3).

Corneal and conjunctival staining can be determined with different dyes: with fluorescein, staining must be graded as quickly as possible after instillation, since the dye then diffuses rapidly into the tissue and its high luminosity blurring the stain margin; staining after lissamine green, persists at high contrast and may therefore be observed for a considerable period.

The presence of corneal staining can be defined as more than one dot of fluorescein staining over the corneal surface (see Figure 4). Superficial punctatae keratitis can be graded according to the Oxford Scheme.24

Lissamine green is available as impregnated strips or as a preprepared solution (see Figure 4). Staining at the interpalpebral region of the nasal and temporal conjunctiva is graded using the oxford scheme too. ${ }^{24}$

The severity designations used for both fluorescein and lissamine green staining are the following: 0 to I, normal; II to III, mild to moderate; and IV to V, severe (see Figure 5). 


\section{Management of Patients with Ocular Surface Disease and Glaucoma}

Obviously, treatment of associated diseases of the anterior surface, such as dry eye, blepharitis, allergy and correction of eyelid abnormalities, should be considered trying to reduce symptoms and signs of OSD. Non-specific treatment of OSD may include non-BAK-preserved tear replacement drops to relieve symptoms.

In fact, previous literature has pointed out that when prescribing glaucoma drops to control one disease (glaucoma), ophthalmologists could be exacerbating another (OSD)..$^{8,11-13}$ The OSD is frequent in glaucoma patients and is not only limited to a small sample of allergic patients, but it is more probably due to a direct toxic effect on eye structures. The toxic effect of BAK on the ocular surface has been widely demonstrated in the literature as being time and dose dependent, ${ }^{8}$ but in 2007 the International Dry Eye Workshop stated that BAK toxic effects might be reversible. ${ }^{15}$ All worldwide available non-BAK-preserved antiglaucoma medications are listed on Table 1 BAK-free drugs are less associated with ocular surface symptoms and signs.

Several papers have demonstrated that ocular surface signs are more prevalent with BAK-preserved glaucoma medications than with nonBAK-preserved drugs. ${ }^{25}$ Moreover, a decrease of all ocular symptoms and signs is observed when preserved eye drops are reduced or changed to preservative free or BAK-free formulations.
In a recent paper, we have examined the corneal status after switching from a BAK-preserved to a BAK-free prostaglandin analogue by in vivo confocal microscopy. ${ }^{26}$

Previously, BAK-preserved treated patients showed an improvement in confocal parameters presenting increased epithelial cells, reduced keratocyte activation, increased number of corneal nerves, decreased number of bead-like formations and nerve tortuosity. Our observations seem to confirm the potential reversibility of toxic effects on ocular surface due to chronic exposure to BAK.

When it is not possible to prescribe a BAK-free therapy, it should be considered to add an artificial tear to reduce symptoms and improve signs.

The reduction of BAK exposition is therefore mandatory considering that the ocular surface status influences glaucoma patient's QOL ${ }^{27}$ and can also impair both adherence to therapy and glaucoma surgery's efficacy.

In conclusion, the main aim of a glaucoma specialist is to cure his or her own patients to prevent disease progression but we must also consider the chance to reduce ocular discomfort (OSD) due to a chronic exposition to topical drugs and preservatives. Long-term exposure to BAK should be minimised preferring non-BAK-preserved or BAK-free glaucoma medications, were available, as well as fixed combinations. Also, treatment of associated diseases of the anterior surface may reduce signs; and use of non-BAK-preserved tears may reduce symptoms
1. AGIS Investigators. Advanced Glaucoma Intervention Study (AGIS): 7. The relationship between control of intraocular pressure and visual field deterioration, Am J Ophthalmol 2000;130:429-40.

2. Leske MC, Heijl A, Hussein M, et al., Factors for glaucoma progression and the effect of treatment: the Early Manifest Glaucoma Trial, Arch Ophthalmol, 2003:121:48-56.

3. Kass MA, Heuer DK, Higginbotham EJ, et al., The Ocular Hypertension Treatment Study: a randomized trial determines Hypertension Treat Hent Study. a randomized trial determines that topical ocular hypotensive medication delays or prevents the onset of primary open-angle glaucoma, Arch Ophthalmol

4. Miglior S, Zeyen T, Pfeiffer N, et al., Results of the European glaucoma prevention study, Ophthalmology, 2005;112:366-75

5. European Glaucoma Society, Terminology and guidelines fo glaucoma. In: European Glaucoma Society Guidelines 3rd ed, Savona, Italy: Editrice Dogma, 2008;117-53.

6. Baudouin C, Labbé A, Liang $\mathrm{H}$, et al., Preservatives in eyedrops: the good, the bad and the ugly, Prog Retin Eye Res, 2010;29:312-34

7. Baudouin C, Detrimental effect of preservatives in eyedrops: implications for the treatment of glaucoma, Acta Ophthalmo , 2008: 86:716-26.

8. Rossi GC, Pasinetti GM, Scudeller L, et al., Risk factors to develop ocular surface disease in treated glaucoma or ocular hypertension patients, Eur $J$ Ophthalmol, 2013:23:296-302.

9. Labbe A, Pauly A, Liang H, et al., Comparison of toxicological profiles of benzalkonium chloride and polyquaternium-1: an experimental study, J Ocul Pharmacol Ther, 2006; 22:267-78 0. Ammar DA, Kahook MY, Effects of glaucoma medications an preservatives on cultured human trabecular meshwork and non pigmented ciliary epithelial cell lines, Br J Ophthalmo 2011; 95:1466-69.

11. Leung EW, Medeiros FA, Weinreb RN, Prevalence of ocular surface disease in glaucoma patients, I Glaucoma, 2008:17:350-5

12. Rossi GC, Tinelli C, Pasinetti GM, et al., Dry eye syndromerelated quality of life in glaucoma patients, Eur J Ophthalmol, $2009: 4 \cdot 572-9$

13. Fechtner RD, Godfrey DG, Budenz D, et al., Prevalence of ocular surface complaints in patients with glaucoma using topical intraocular pressure-lowering medications, cornea, topical intraocular pr $29: 618-21$.

2010; 29:618-21.
14. Rolando M, Geerling G, Dua HS, et al., Emerging treatment paradigms of ocular surface disease: proceedings of the Ocular Surface Workshop, Br J Ophthalmol 2010:94(Suppl.1):i1-i9.

15. Report of the International Dry Eye Workshop (DEWS). The epidemiology dry eye disease, Ocul Surf, 2007:5:27-41.

16. Moss SE, Klein R, Klein BE, Prevalence of and risk factors for dry eye syndrome, Arch Ophthalmol, 2000:118:1264-8.

17. Tsai $\amalg H$, Derby E, Holland EJ, Khatana AK, Incidence and prevalence of glaucoma in severe ocular surface disease, cornea, 2006;25:530-2.

18. Begley $C G$, Chalmers RL, Abetz $L$, et al., The relationship between habitual patient-reported symptoms and clinical signs among patients with dry eye of varying severity, Invest ophthalmol Vis Sci, 2003;44:4753-61.
19. Nichols KK, Nichols JJ, Mitchell GL, The lack of associatio between signs and symptoms in patients with dry eye disease, Cornea, 2004;23:762-70.

20. Atkinson MJ, Stewart WC, Fain JM, et al., A new measure of patient satisfaction with ocular hypotensive medications: the Treatment Satisfaction Survey for Intraocular Pressure (TSSIOP), Health Qual Life Outcomes, 2003;1:67.

21. Lee BL, Gutierrez P, Gordon M, et al., The Glaucoma symptom scale. A brief index of glaucoma specific symptoms, Arch Ophthalmol, 1998:116:861-6.

22. Ruiz MA, Pardo A, Martinez de la Casa JM, et al., Cultura adaptation and validation into Spanish of the Glaucoma Symptom Scale, Med Clin, 2009:132:89-97.

23. Rossi GC, Pasinetti GM, Scudeller L, et al., The Italian version of the Glaucoma Symptom Scale questionnaire: Translation Validation and Reliability, I Glaucoma, 2013;22:44-51.

24. Bron AJ, Evans VE, Smith JA, Grading of corneal and conjunctival staining in the context of other dry eye tests, Cornea, 2003;22:640-9.

25. Jaenen N, Baudouin C, Pouliquen P, et al., Ocular symptoms and signs with preserved and preservative-free glaucoma medications, Eur J Ophthalmol, 2007;17:341-9.

26. Rossi GC, Blini M, Scudeller L, et al., Effect of preservativefree Tafluprost on keratocytes, sub-basal nerves, and endohelium: a single blind one-year confocal study on naïve er treated ga or treated glaucoma and hypertensive patients versus a

27. Rossi G, Pasinetti GM, Scudeller L, Bianchi PE, Ocular surface disease and glaucoma: how to evaluate impact on quality of disease and glaucoma: how to evaluate im
life, J Ocul Pharmacol Ther, 2013;29:390-4. 